\title{
AVALIAÇÃO DA QUALIDADE DOS REGISTROS DE ENFERMAGEM NOS CUIDADOS PÓS-OPERATÓRIOS IMEDIATOS
}

\author{
A quality assessment of immediate postoperative nursing care documentation
}

\section{Evaluación de la calidad de los registros de enfermería en los cuidados postoperatorios inmediatos}

\author{
Emanuela Batista Ferreira e Pereira ${ }^{1 *}$, Gedalva Pereira de Lima², Heloise Agnes Gomes Batista da Silva ${ }^{3}$, \\ Kássia Maria da Hora Teixeira ${ }^{4}$, Brenna Cavalcanti Maciel Modesto ${ }^{5}$, Magdala de Araújo Novaes ${ }^{6}$
}

RESUMO: Objetivo: Avaliar a qualidade dos registros de enfermagem nos cuidados pós-operatórios imediatos em unidades de referência no estado de Pernambuco. Método: Estudo transversal, descritivo, documental e comparativo com abordagem quantitativa, realizado em três hospitais (A, B, C). A amostra foi composta por 130 prontuários no período de outubro a novembro de 2015 com dados coletados por meio de um formulário semiestruturado. Resultados: Os registros, a checagem e as avaliações dos procedimentos foram feitos de formas diferentes nos hospitais avaliados. No hospital A não houve registro dos sinais vitais, enquanto nos hospitais B e C, os mesmos foram verificados. Sobre a escala de Aldrete e Kroulik (AK), nas instituições A e C não houve realização do cálculo em 100\% dos casos. As três instituições apresentaram bons resultados, como prontuários legíveis e sem a presença de rasuras. Conclusão: Os hospitais analisados não atendem aos padrões preconizados pelas principais literaturas na área de cuidados pós-operatórios quanto aos registros de enfermagem.

Palavras-chave: Qualidade da assistência à saúde. Registros de enfermagem. Cuidados pós-operatórios. Enfermagem de centro cirúrgico.

ABSTRACT: Objective: To assess the quality of immediate postoperative nursing care documentation in reference units in the state of Pernambuco, Brazil. Method: A descriptive, cross-sectional, documental and comparison study with a qualitative approach performed in three hospitals (A, B, C). The sample was comprised of 130 health records from October to November 2015 and the data was collected from a semi-structured form. Results: The records, checking and evaluation of procedures were performed differently in each of the assessed hospitals. In hospital A, there was no recording of vital signs, whereas in hospitals B and C, there was. In institutions A and C, in 100\% of the cases, there was no calculation performed that used the Aldrete-Kroulik scale. The three institutions demonstrated good results with legible documentation that did not have many erased items. Conclusion: It was found that the hospitals analyzed don't meet the standards proposed by the predominant postoperative care literature regarding nursing documentation.

Keywords: Quality of health care. Nursing records. Postoperative care. Operating room nursing.

\footnotetext{
'Enfermeira; doutoranda em Cirurgia pelo Programa de Pós-graduação em Cirurgia pela Universidade Federal de Pernambuco (UFPE); professora assistente na Faculdade de Enfermagem Nossa Senhora das Graças (FENSG) da Universidade de Pernambuco (UPE) - Recife (PE), Brasil.

2Enfermeira pela FENSG da UPE; pós-graduanda em Unidade de Terapia Intensiva pelo Instituto de Desenvolvimento Educacional (IDE); enfermeira da Emergência do Hospital Miguel Arraes - Recife (PE), Brasil. ${ }^{3}$ Enfermeira pela FENSG da UPE; residente em Terapia Intensiva no Instituto de Medicina Integral Professor Fernando Figueira (IMIP) pela Secretaria Estadual de Saúde de Pernambuco - Recife (PE), Brasil.

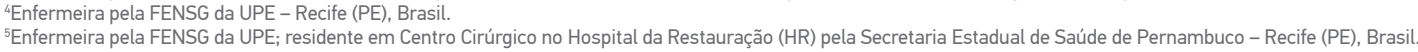

${ }^{6}$ Cientista da Computação; doutora em Bioinformatique pela Universidade Aix-Marseille Il; professora associada do Departamento de Medicina Clínica da UFPE; coordenadora do Núcleo de Telessaúde do

Hospital das Clínicas da UFPE - Recife (PE), Brasil.

*Autor correspondente: emanuela.pereira@upe.br

Recebido: 25/09/2017 - Aprovado: 05/01/2018

DOI: $10.5327 / 21414-4425201800010005$
} 
RESUMEN: Objetivo: Evaluar la calidad de los registros de enfermería en el cuidado postoperatorio inmediato en las unidades de referencia en el Estado de Pernambuco, Brazil. Métodos: Estudio transversal, descriptivo, documental y comparativo con enfoque cuantitativo realizado en tres hospitales (A, B, C). La muestra estuvo compuesta por 130 registros médicos durante el período de octubre a noviembre de 2015 con los datos recolectados a través de un formulario semi-estructurado. Resultados: Los registros, el control y la evaluación de procedimientos fueron hechos de diferentes maneras en los hospitales evaluados. En el hospital A, no hubo registro de signos vitales, mientras que en los hospitales $\mathrm{B}$ y $\mathrm{C}$, han sido verificados. En la escala de Aldrete y Kroulik, en las instituciones A y C, no había ninguna realización del cálculo en el 100\% de los casos. Las tres instituciones mostraron buenos resultados en lectura de cartas y la presencia de raspaduras. Conclusión: Parece que los hospitales analizados no cumplen con las normas recomendadas por las principales literaturas en el área de cuidados postoperatorios acerca de los registros de enfermería. Palabras clave: Calidad de la atención de salud. Registros de enfermería. Cuidados posoperatorios. Enfermería de quirófano.

\section{INTRODUÇÃO}

As anotações de enfermagem são documentos primordiais das ações e atividades desempenhadas pela equipe de enfermagem na prática profissional, comprovam a realização do cuidado e garantem a qualidade da assistência. São caracterizadas como registros que se destinam a toda a equipe de enfermagem e são imprescindíveis para a execução da sistematização da assistência de enfermagem (SAE). Devem avaliar e refletir o paciente de forma complexa, envolvendo especialmente aspectos biopsicossociais, que permitam a evolução do caso e a elaboração de estratégias que visam à integralidade do cuidado ${ }^{1}$.

Esses registros garantem melhor comunicação entre a equipe de saúde e são utilizados como ferramentas ético-legais para evidenciar negligências por parte dos profissionais e da própria instituição hospitalar, contribuindo para uma avaliação da qualidade do serviço ${ }^{2}$. Para gerenciar a qualidade da assistência de enfermagem e os gastos decorrentes com a prestação dos serviços, a auditoria tem sido o instrumento mais utilizado ${ }^{3}$.

A auditoria na área da saúde surgiu com o propósito de avaliar a qualidade da assistência prestada ao paciente. É um método que avalia de forma sistemática e independente os fatos obtidos por meio da observação e mensuração das recomendações previstas nas normas vigentes, com a finalidade de determinar se as ações de saúde e seus resultados estão de acordo com os indicadores de qualidade ${ }^{3}$.

Assim, a avaliação da qualidade do serviço prestado pela equipe de enfermagem reflete a análise dos registros identificados pela auditoria. A ausência dos registros promove diminuição na continuidade do cuidado e nos setores de alta complexidade, como a sala de recuperação pós-anestésica (SRPA). O enfermeiro deve assegurar que o paciente seja avaliado de forma integral, especialmente quanto ao registro dos sinais vitais e à presença de intercorrências ${ }^{4}$.

A SRPA é um ambiente que apresenta rotinas peculiares no atendimento aos pacientes, sendo caracterizado por uma assistência crítica, que tem como meta a prestação de cuidados intensivos no pós-operatório imediato (POI) dos pacientes que são submetidos a procedimentos anestésico-cirúrgicos e diagnósticos ${ }^{4,5}$.

Essa unidade hospitalar é destinada aos pacientes desde o término do procedimento até a estabilidade dos sinais vitais e do nível de consciência, com a finalidade de detectar e evitar complicações que possam ocorrer em consequência da anestesia ou do ato cirúrgico 5 .

A relevância da temática é corroborada pela necessidade dos registros de enfermagem nos prontuários e, particularmente, pelos cuidados diante da monitorização dos sinais vitais e das intervenções de enfermagem. As lacunas de pesquisas sobre qualidade de registros na SRPA merecem atenção mediante a evidência que tais registros representam para qualificação das ações e práticas exercidas pela equipe de enfermagem.

\section{OBJETIVO}

Avaliar a qualidade dos registros de enfermagem nos cuidados pós-operatórios imediatos em unidades de referência no estado de Pernambuco, Brasil.

\section{MÉTODO}

Estudo transversal, descritivo, documental e comparativo, com abordagem quantitativa, que realizou uma avaliação na qualidade dos registros de enfermagem na SRPA em unidades de referências no estado de Pernambuco. 
Foi escolhida a abordagem quantitativa, por corresponder à etapa do processo de análise descritiva, que permite a exploração de dados para uma aproximação da realidade estudada, na procura de algum padrão ou comportamento relevante que esteja presente no conjunto de dados ${ }^{6}$.

A coleta de dados foi realizada no período de outubro a novembro de 2015, na SRPA de três unidades hospitalares de referência na cidade de Recife, Pernambuco, Brasil. As instituições são aqui designadas hospital A, hospital B e hospital C, dispondo de 16, 7 e 9 leitos, respectivamente.

Os critérios de inclusão foram prontuários de pacientes internados nessas unidades de cuidados POI, submetidos a procedimentos cirúrgicos eletivos, com idades acima dos 18 anos, de ambos os sexos e com a permanência mínima de 2 horas na SRPA. Sob esses critérios, a amostra do estudo foi constituída de 130 prontuários, sendo 50 do hospital A, 50 do hospital B e 30 do hospital C.

Para coleta dos dados, foi utilizado um formulário semiestruturado composto de três partes. Por meio desse impresso, foi efetivada a identificação dos dados de admissão registrados pela enfermagem, a caracterização dos registros de enfermagem relacionados à monitorização hemodinâmica, o índice de Aldrete e Kroulik (AK) e a identificação das principais intercorrências e ações registradas pela enfermagem nas unidades de referência.

A parte I do instrumento compreendeu oito questões objetivas e subjetivas, as quais contemplaram a qualidade dos registros de enfermagem quanto aos aspectos epidemiológicos e clínicos, como sexo, idade, hipótese diagnóstica, alergias, especialidade cirúrgica, tipo da anestesia e antecedentes clínicos $(\mathrm{AC})^{7}$.

A parte II compreendeu o registro da monitorização hemodinâmica e sinais vitais (SSVV) e padrões como pressão arterial (PA), frequência cardíaca (FC), saturação de oxigênio $\left(\right.$ Sat $\left.\mathrm{O}_{2}\right)$, frequência respiratória (FR) e temperatura $(\mathrm{T})^{7}$.

A parte III compreendeu os registros das intercorrências apresentadas durante a permanência na SRPA e os cuidados de enfermagem aplicados a cada intercorrência. Reforça-se que o instrumento de coleta de dados baseou-se em estudos similares, sofrendo algumas alterações e adaptações ${ }^{7}$.

Para análise dos dados, foi construído um banco de dados em planilha eletrônica Microsoft Excel ${ }^{\circledR}$, o qual foi exportado para o software Statistical Package for the Social Sciences (SPSS). Para avaliar a qualidade dos registros de enfermagem quanto ao perfil epidemiológico e clínico dos prontuários avaliados em cada hospital, foram calculadas as frequências percentuais e distribuições de frequência.
Foi realizado, ainda, o levantamento dos fatores relacionados à monitorização hemodinâmica e SSVV dos pacientes, dos fatores relacionados ao registro de admissão e ações de enfermagem, dos registros das intercorrências e das intervenções de enfermagem realizadas, para cada instituição avaliada. Para comparação dos fatores avaliados entre as instituições, foi aplicado o teste do $\chi^{2}$ para homogeneidade. Todas as análises foram realizadas considerando o nível de significância de 5\%.

O estudo foi aprovado pelo Comitê de Ética em Pesquisa da Universidade de Pernambuco (CEP/UPE), atendendo à Resolução $n^{\circ}$ 466/2012, do Conselho Nacional de Saúde (número do parecer 1.265.296). Para a presente pesquisa, não se fez necessária a utilização do Termo de Consentimento Livre e Esclarecido (TCLE), pois se trata de uma investigação documental.

\section{RESULTADOS}

Na Tabela 1, observa-se a distribuição do perfil epidemiológico e clínico dos pacientes, segundo cada hospital avaliado.

Ao observar o teste de homogeneidade, verificou-se que ele é significativo em todos os fatores (valor $\mathrm{p}=0,002$ para sexo e valor $\mathrm{p}<0,001$ para os demais), indicando que existe diferença da prevalência destes fatores nos hospitais avaliados.

A distribuição dos fatores relacionados à monitorização hemodinâmica dos pacientes está demonstrada na Tabela 2.

O teste de homogeneidade da distribuição dos fatores relacionados à monitorização hemodinâmica e aos sinais vitais, entre os hospitais avaliados, foi significativo em todos os fatores (valor $\mathrm{p}<0,001$ ), indicando que os registros, a checagem e as avaliações dos procedimentos são feitos de formas diferentes nas três instituições, tendo o hospital A os resultados mais distantes do preconizado pela literatura.

Na Tabela 3, destaca-se a distribuição dos fatores relacionados aos registros de admissão e às ações de enfermagem, segundo o hospital avaliado.

O teste de homogeneidade da distribuição dos fatores relacionados aos registros e às ações de enfermagem nos três hospitais avaliados foi significativo em todos os fatores, (valor $\mathrm{p}<0,05$ ), indicando que os registros de admissão e ações de enfermagem são feitos de forma diferenciada entre os hospitais A, B e C, tendo o último hospital melhor situação dos 
Tabela 1. Distribuição dos registros de enfermagem quanto ao perfil epidemiológico e clínico dos pacientes, segundo o hospital.

\begin{tabular}{l|c|c|c|c|}
\multirow{2}{*}{ Variáveis } & \multicolumn{3}{|c|}{ Hospital } & \multirow{2}{*}{ Valor } \\
\cline { 2 - 4 } & A & B & C & P
\end{tabular}

\begin{tabular}{|c|l|l|l|l|}
\hline Sexo n (\%) & & & \\
\cline { 1 - 3 } Masculino & $35(70,0)$ & $18(36,0)$ & $19(63,3)$ & $0,002^{*}$ \\
\cline { 1 - 3 } Feminino & $15(30,0)$ & $32(64,0)$ & $11(36,7)$ & \\
\hline
\end{tabular}

Idade (anos)

\begin{tabular}{|l|c|c|c|c|}
\hline Mínimo & 19 & 18 & 34 & - \\
\hline Máximo & 87 & 81 & 84 & - \\
\hline Média $\pm D P$ & $48,0 \pm 18,5$ & $56,5 \pm 16,3$ & $60,9 \pm 13,0$ & - \\
\hline
\end{tabular}

Especialidade cirúrgica n (\%)

\begin{tabular}{|c|c|c|c|}
\hline Bucomaxilofacial & $3(6,0)$ & - & - \\
\hline Cardiovascular & - & - & $28(93,3)$ \\
\hline Geral & $7(14,0)$ & $14(28,0)$ & - \\
\hline Neurocirurgia & $13(26,0)$ & - & - \\
\hline Oncologia & $5(10,0)$ & $23(46,0)$ & - \\
\hline Ortopedia & $13(26,0)$ & - & - \\
\hline Plástica & $2(4,0)$ & $1(2,0)$ & - \\
\hline Urologia & - & $12(24,0)$ & - \\
\hline Vascular & $7(14,0)$ & - & $2(6,7)$ \\
\hline
\end{tabular}

Tipo de anestesia $\mathrm{n}(\%)$

\begin{tabular}{|c|c|c|c|c|}
\hline Bloqueio & $4(6,7)$ & - & - & \\
\hline Sedação & $8(13,3)$ & $6(9,0)$ & - & \\
\hline Geral & $17(28,3)$ & $23(34,3)$ & $27(90,0)$ & \\
\hline $\begin{array}{l}\text { Geral } \\
\text { balanceada }\end{array}$ & $9(15,0)$ & $6(9,0)$ & $2(6,7)$ & \\
\hline Local & $1(1,7)$ & $3(4,5)$ & $1(3,3)$ & \\
\hline Peridural & $3(5,0)$ & $11(16,4)$ & - & \\
\hline Raquianestesia & $14(23,3)$ & $17(25,4)$ & - & \\
\hline Venosa total & $4(6,7)$ & $1(1,5)$ & - & \\
\hline \multicolumn{5}{|l|}{ Alergias n (\%) } \\
\hline Sim & $8(16,0)$ & $12(24,0)$ & $2(6,7)$ & \multirow{3}{*}{$<0,001^{*}$} \\
\hline Não & $27(54,0)$ & $38(76,0)$ & $28(93,3)$ & \\
\hline Não informado & $15(30,0)$ & - & - & \\
\hline \multicolumn{5}{|c|}{ Antecedentes clínicos n (\%) } \\
\hline Sim & $25(50,0)$ & $33(66,0)$ & $28(93,3)$ & \multirow{3}{*}{$<0,001^{*}$} \\
\hline Não & $10(20,0)$ & $17(34,0)$ & $2(6,7)$ & \\
\hline Não informado & $15(30,0)$ & - & - & \\
\hline
\end{tabular}

DP: desvio padrão; *valor do teste do $\chi^{2}$ para homogeneidade (se valor $p<0,05$ a distribuição do fator avaliado é idêntica nos hospitais avaliados). seus registros, exceto no quesito intercorrências e intervenções de enfermagem, em que houve resultados de ausência de notificações em todos os hospitais.

\section{DISCUSSÃO}

Diante dos dados obtidos, foi observada uma predominância de homens admitidos na SRPA dos hospitais A e C; apenas a instituição $B$ registrou um quantitativo maior de mulheres. O predomínio do sexo masculino no presente estudo diverge da literatura, pois em estudo realizado com prontuários de

Tabela 2. Distribuição dos registros de enfermagem quanto aos fatores relacionados a monitorização hemodinâmica e sinais vitais dos pacientes, segundo o hospital.

\begin{tabular}{|c|c|c|c|c|}
\hline \multirow{3}{*}{$\begin{array}{l}\text { Fator } \\
\text { avaliado }\end{array}$} & \multicolumn{3}{|c|}{ Hospital } & \multirow{3}{*}{$\begin{array}{c}\text { Valor } \\
\mathbf{p}\end{array}$} \\
\hline & A & B & C & \\
\hline & n (\%) & n (\%) & n (\%) & \\
\hline
\end{tabular}

Registro dos sinais vitais

\begin{tabular}{l|c|c|c|c|}
\cline { 1 - 3 } Sim & $20(40,0)$ & $39(78,0)$ & $30(100,0)$ & $<0,001$ * \\
\cline { 1 - 3 } & $30(60,0)$ & $11(22,0)$ & - &
\end{tabular}

Verificação do registro de frequência cardíaca

\begin{tabular}{|c|c|c|c|c|}
\hline Sim & $14(28,0)$ & $39(78,0)$ & $30(100,0)$ & \\
\hline Não & $36(72,0)$ & $11(22,0)$ & - & \\
\hline
\end{tabular}

Verificação do registro de frequência respiratória

\begin{tabular}{l|l|l|c|l} 
Sim & $12(24,0)$ & $38(76,0)$ & $30(100,0)$ & $<0,001$ * \\
\cline { 1 - 3 } Não & $38(76,0)$ & $12(24,0)$ & - &
\end{tabular}

Verificação do registro de pressão arterial

\begin{tabular}{|c|c|c|c|c|}
\hline Sim & $15(30,0)$ & $39(78,0)$ & $30(100,0)$ & \\
\hline Não & $35(70,0)$ & $11(22,0)$ & - & \\
\hline
\end{tabular}

Verificação do registro de saturação de oxigênio

\begin{tabular}{|c|c|c|c|c|}
\hline Sim & $14(28,0)$ & $38(76,0)$ & $30(100,0)$ & \\
\hline Não & $36(72,0)$ & $12(24,0)$ & - & \\
\hline
\end{tabular}

Verificação do registro de temperatura

\begin{tabular}{|c|c|c|c|}
\hline Sim & - & $6(12,0)$ & $30(100,0)$ \\
\hline Não & $50(100,0)$ & $44(88,0)$ & - \\
\hline
\end{tabular}

Aplicação Índice de Aldrete e Kroulik

\begin{tabular}{|c|c|c|c|}
\hline Sim & - & $15(30,0)$ & - \\
\hline Não & $50(100,0)$ & $35(70,0)$ & $30(100,0)$ \\
\hline
\end{tabular}

Avaliação correta do Aldrete e Kroulik

\begin{tabular}{l|l|l|} 
Sim & - & $6(40,0)$ \\
\hline Não & - & $9(60,0)$ \\
\hline
\end{tabular}

*Valor $p$ do teste do $\chi^{2}$ para homogeneidade (se valor $p<0,05$ a distribuição do fator avaliado é idêntica nos hospitais avaliados). 
260 pacientes cirúrgicos admitidos na SRPA, mais de 50\% pertenciam ao sexo feminino ${ }^{4}$.

As intervenções cirúrgicas realizadas predominantemente nas instituições foram neurocirurgias, cirurgias ortopédicas, oncológicas e cardiovasculares. Quando associadas às variáveis tipo de cirurgia e sexo, a Política Nacional de Atenção à Saúde do Homem aponta maior exposição aos acidentes por causas externas, bem como a aversão desses indivíduos ao autocuidado e à prevenção de morbidades, obrigando, assim, o sistema a assisti-los nas fases mais avançadas das doenças e tratamentos ${ }^{8}$.

Observou-se que a anestesia geral foi a mais utilizada nas instituições. É de grande importância o registro do tipo de anestesia usada no ato cirúrgico, pois por meio da efetiva e correta monitorização é possível identificar, em tempo hábil, as alterações apresentadas no período pós-anestésico e, consequentemente, o entendimento dos fármacos ${ }^{4}$.

Para propiciar a segurança do paciente, é imprescindível registrar as alergias no prontuário, pois identificar corretamente e checar a prescrição antes de fazer a administração

Tabela 3. Distribuição dos fatores relacionados à qualidade dos registros de enfermagem, segundo o hospital avaliado.

\begin{tabular}{|c|c|c|c|c|}
\hline \multirow{3}{*}{$\begin{array}{l}\text { Fator } \\
\text { avaliado }\end{array}$} & \multicolumn{3}{|c|}{ Hospital } & \multirow{3}{*}{$\begin{array}{c}\text { Valor } \\
\text { p }\end{array}$} \\
\hline & $\mathbf{A}$ & B & C & \\
\hline & n (\%) & n (\%) & n (\%) & \\
\hline \multicolumn{5}{|c|}{ Prontuário legível } \\
\hline Sim & $33(66,0)$ & $35(70,0)$ & $30(100,0)$ & $0,002^{*}$ \\
\hline Não & $17(34,0)$ & $15(30,0)$ & - & \\
\hline \multicolumn{5}{|c|}{ Prontuário com rasuras } \\
\hline Sim & $11(22,0)$ & $19(38,0)$ & - & $<0,001^{*}$ \\
\hline Não & $39(78,0)$ & $31(62,0)$ & $30(100,0)$ & \\
\hline \multicolumn{5}{|c|}{ Registros assinados e rubricados } \\
\hline $\operatorname{Sim}$ & $32(64,0)$ & $23(46,0)$ & $30(100,0)$ & $<0,001^{*}$ \\
\hline Não & $18(36,0)$ & $27(54,0)$ & - & \\
\hline \multicolumn{5}{|c|}{ Registro do horário das intervenções } \\
\hline Sim & $8(16,0)$ & $39(78,0)$ & $30(100,0)$ & $<0,001^{*}$ \\
\hline Não & $42(84,0)$ & $11(22,0)$ & - & \\
\hline \multicolumn{5}{|c|}{ Registros das intercorrências } \\
\hline Sim & $3(6,0)$ & $5(10,0)$ & $8(26,7)$ & $0,020^{*}$ \\
\hline Não & $47(94,0)$ & $45(90,0)$ & $22(73,3)$ & \\
\hline \multicolumn{5}{|c|}{ Registro da intervenção de enfermagem } \\
\hline Sim & $1(2,0)$ & $3(6,0)$ & $7(23,3)$ & $0,003^{*}$ \\
\hline Não & $49(98,0)$ & $47(94,0)$ & $23(76,7)$ & \\
\hline
\end{tabular}

*Valor $p$ do teste do $\chi^{2}$ para homogeneidade (se valor $p<0,05$ a distribuição do fator avaliado é idêntica nos hospitais avaliados). medicamentosa ameniza os riscos de reações alérgicas e intercorrências. Nos hospitais B e C, houve registro de alergias em todos os prontuários analisados; porém, na instituição A havia falta dessas informações em $30 \%$ dos prontuários, demonstrando uma fragilidade da assistência ${ }^{9,10}$.

Quanto aos antecedentes clínicos, notou-se que em parcela significativa dos prontuários do hospital A não constavam as comorbidades dos pacientes. É no histórico de enfermagem que as informações dos pacientes são coletadas, com o objetivo de avaliar os antecedentes pessoais e familiares, bem como analisar evidências e fatores de risco para a saúde global do paciente ${ }^{11}$. A falta dessas informações expressa uma vulnerabilidade para os pacientes e aumenta os riscos de complicações no pós-operatório ${ }^{12}$.

A faixa etária dos pacientes nos três hospitais estudados mostrou média de idade predominante adulta. Os achados de estudos no que diz respeito à variável idade afirmam que é preciso assistir os pacientes de faixa etária avançada com mais cautela, pois as morbidades e o processo de envelhecimento aumentam o risco de complicações no pós-operatório, devido ao desequilíbrio das funções basais. Em um estudo de revisão integrativa foram investigadas as complicações durante o período pós-anestésico e evidenciaram-se lacunas quanto ao mecanismo de verificação de complicações e aos manejos de enfermagem. É fundamental, então, o registro dessa informação para planejar a assistência em todo o pós-operatório e identificar problemas precocemente ${ }^{13,14}$.

De acordo com a Associação Brasileira de Enfermeiros de Centro-Cirúrgico, Recuperação Anestésica e Centro de Material de Esterilização (SOBECC) ${ }^{15}$, a monitoração hemodinâmica e de SSVV é imprescindível durante a permanência do paciente na SRPA e se faz necessária a avaliação por meio da escala de AK para efetivação da alta do paciente. A SRPA é um local onde os pacientes recebem cuidados intensivos e tem como objetivo maior prevenir e detectar precocemente possíveis complicações póscirúrgicas, enfatizando a estabilidade dos sinais vitais e o retorno da consciência ${ }^{16}$.

As instituições estudadas apresentaram diferentes resultados quanto à verificação hemodinâmica e à checagem dos sinais vitais. Não foi observado consenso quanto à mensuração de alguns parâmetros, sendo identificada, inclusive, a ausência do registro de itens, como a temperatura, em sua totalidade.

Durante a entrada e permanência dos pacientes na SRPA, principalmente no POI, período de maior incidência de 
complicações, é preciso que o enfermeiro realize exame físico adequado, estabilize os SSVV e avalie o nível de consciência, assegurando que o paciente seja visto de forma integral de acordo com o ato cirúrgico, agentes anestésicos utilizados e riscos potenciais ${ }^{4}$.

O enfermeiro deve sempre estar atento à monitorização hemodinâmica. A verificação da FR é fundamental, pois é uma função afetada de forma significativa pela ação residual de bloqueadores neuromusculares encontrados nos anestésicos. A instabilidade do sistema cardiovascular exige atenção para a FC, pois a hipotensão é um dos achados mais comuns, possuindo diferentes causas, como a vasodilatação e a diminuição do débito cardíaco ${ }^{4,16}$.

Deve ser realizada, também, a monitorização da PA rigorosamente, pois o seu aumento poderá estar relacionado à dor no POI, distensão vesical, agitação neuromuscular, entre outros motivos. A mensuração da Sat $\mathrm{O}_{2}$ deve sempre ser avaliada, considerando que a função respiratória é afetada, de forma significativa, devido à ação residual dos anestésicos utilizados ${ }^{4,16}$.

É importante também a avaliação do sistema termorregulador, pois a $\mathrm{T}$ poderá variar para valores mais altos ou mais baixos. A hipotermia é um dos achados de maior prevalência e pode ser consequência de um retardo no despertar ou maior permanência hospitalar ${ }^{4,16}$.

A escala de AK avalia parâmetros como atividade muscular, respiração, circulação, consciência e Sat $\mathrm{O}_{2}$, sendo uma escala simples, eficaz e de fácil aplicação. A aplicação dessa escala é utilizada como base para os critérios de alta do paciente da SRPA. Negligenciar esses critérios acarreta riscos à estabilidade hemodinâmica do paciente ${ }^{16,17}$.

No item realização da escala de AK, nos hospitais A e C não houve realização do cálculo na totalidade dos casos. No hospital B foi realizado o cálculo de AK; no entanto, a avaliação dos pacientes quanto ao percentual expressivo foi incorreta. Nas instituições avaliadas houve uma amostra preocupante quanto à deficiência na utilização do instrumento para critério de alta, o qual se pode inferir que poderá contribuir para a morbimortalidade dos pacientes.

Concomitante a tais evidências, a SRPA é um local caracterizado por um atendimento de alta complexidade, e a ausência dos registros de enfermagem nesse cenário pode causar danos aos pacientes assistidos no setor e prejuízos para a instituição de saúde, minimizando a segurança da assistência realizada pelo enfermeiro e pela sua equipe ${ }^{18}$.

As três instituições analisadas apresentaram bons resultados nos itens prontuários legíveis e presença de rasuras. Tais resultados estão em consonância com o que preconiza a Resolução do Conselho Federal de Enfermagem (COFEn) no 311/2007, o Código de Ética dos Profissionais de Enfermagem. Em seu escopo, está descrito que os registros de enfermagem devem ser realizados de forma objetiva e legível, garantindo a identificação correta das informações por outros profissionais de saúde ${ }^{19}$.

A ausência de registros dificulta a identificação do profissional, do procedimento ou da prescrição realizada, como também, se as alterações apresentadas foram observadas durante o período de permanência do paciente na SRPA ${ }^{19}$.

As limitações encontradas no estudo se referem ao número reduzido de evidências sobre a temática, bem como às dificuldades de acesso aos prontuários e à compreensão das anotações e registros efetuados pela equipe de enfermagem na SRPA.

\section{CONCLUSÃO}

Os achados deste estudo permitiram concluir que as práticas de registro de enfermagem desempenhadas nos hospitais analisados não estão em consonância com os padrões preconizados pela literatura na área dos cuidados pós-operatórios.

Há déficit nos registros dos três hospitais avaliados. O déficit nos registros da monitorização hemodinâmica e do índice de recuperação anestésica, por meio da escala de $\mathrm{AK}$, por exemplo, são condições propícias à falha de procedimentos e qualidade da assistência e comprometem a avaliação e a classificação da gravidade e do estado geral do paciente.

Ressalta-se que a ausência dos registros de intervenção realizada pelos profissionais promove um desconhecimento do processo saúde-doença, como também dificulta a comprovação dos procedimentos clínico-cirúrgicos realizados no paciente.

Por fim, o enfermeiro, como líder da equipe de enfermagem, deve estar atento à realização dos registros como forma de garantir a integralidade do cuidado, norteando o plano de assistência de enfermagem durante a permanência do paciente na SRPA e resguardando-se de possíveis sanções ético-legais.

Recomenda-se, assim, a realização de estudos que busquem caracterizar os registros e as anotações de enfermagem, bem como compreender as particularidades e prioridades da área de enfermagem pós-operatória. 


\section{REFERÊNCIAS}

1. Seignemartin BA, de Jesus LR, Vergílio MST, Silva EM. Evaluation of the quality of nursing records in the emergency department of a teaching hospital. Rev Rede Enferm Nord [Internet]. 2013 [acesso em 2015 dez 8];14(6):1123-32. Disponível em: http:// www.revistarene.ufc.br/revista/index.php/revista/article/ viewFile/1352/pdf_1

2. Maziero VG, Vannuchi MTO, Haddad M do CL, Vituri DW, Tada CN. Quality control of patients' monitoring records in a university hospital. Reme Rev Min Enferm [Internet]. 2013 [acesso em 2015 dez 8];17(1):171-6. Disponível em: http:// www.reme.org.br/content/imagebank/pdf/en_v17n1a14.pdf DOI: 10.5935/1415-2762.20130014

3. Valença CN, de Azevedo LMN, Oliveira AG, de Medeiros SSA, Malveira FAS, Germano RM. The scientific literature on nursing audit and quality of records. Rev Pesqui Cuid Fundam [Internet]. 2013 [acesso em 2015 dez 8];5(5):69-76. Disponivel em: http://www.seer. unirio.br/index.php/cuidadofundamental/article/view/1617/pdf_995 DOI: $10.9789 / 2175-5361.2013 v 5 n 5 e s p 69$

4. Cecílio AAS, Peniche A de CG, Popov DCS. Analysis of blood pressure records at post-anesthesia recovery room. Acta Paul Enferm. 2014;27(3):249-54. http://dx.doi.org/10.1590/1982-0194201400042

5. de Lima LB, Rabelo ER. Nursing workload in the postanesthesia care unit. Acta Paul Enferm. 2013;26(2):1 16-22. http://dx.doi.org/10.1590/ S0103-21002013000200003

6. Medronho RA. Epidemiologia. 2a ed. São Paulo: Atheneu; 2008.

7. de Cunha ALSM, Peniche A de CG. Content validity of an instrument to document recovery of patients in post anesthesia care unit. Acta Paul Enferm. 2007;20(2):151-60. http://dx.doi.org/10.1590/ S0103-21002007000200007

8. Brasil. Ministério da Saúde. Política Nacional de Atenção Integral à Saúde do Homem: princípios e diretrizes [Internet]. Brasília: Ministério da Saúde; 2009 [acesso em 2015 dez 8]. Disponível em: http://bvsms.saude.gov.br/bvs/publicacoes/politica_nacional_ atencao_homem.pdf

9. Miranda TMM, Petriccione S, Ferracini FT, Borges Filho WM. Interventions performed by the clinical pharmacist in the emergency department. Einstein (São Paulo). 2012;10(1):74-8. http://dx.doi. org/10.1590/S1679-45082012000100015

10. Brasil. Ministério da Saúde (MS). Protocolo de Segurança na prescrição, uso e administração de medicamentos. Anexo 3 da Portaria MS no 2.095, de 24 de setembro de 2013. 2013 [acesso em 2015 dez 8]. Disponível em: http://www.hospitalsantalucinda.com.br/downloads/ prot_meficamentos.pdf
11. Tavares TS, de Castro AS, Figueiredo ARFF, dos Reis DC. Evaluation of the implementation of the systematic organization of nursing care in a pediatric ward. Rev Min Enferm [Internet]. 2013 [acesso em 2015 dez 8];17(2):42-50. Disponível em: http://www.reme.org.br/artigo/detalhes/650 http://www.dx.doi.org/10.5935/1415-2762.20130022

12. de Oliveira JMA, Silva AMF, Cardoso S de B, Lima FF, Zierer M de S, Carvalho ML. Postoperative complications of cardiovascular surgery with cardiopulmonary by-pass. Rev Interdiscip. 2015 [acesso em 2015 dez 8];8:9-15. Disponível em: http://revistainterdisciplinar. uninovafapi.edu.br/index.php/revinter/article/view/584/pdf_177

13. Giordani AT, Sonobe HM, Ezaias GM, Valério MA, Barra MR, Stadler DV. Profile of surgical patients treated in a public hospital. Rev Enferm UFPE on line [Internet]. 2015 [acesso em 2015 dez 8];9(1):54-61. Disponível em: https://periodicos. ufpe.br/revistas/revistaenfermagem/article/view/10306/10974 DOI: 10.5205/reuol.6817-60679-1-ED.0901201508

14. Ribeiro M, Peniche A de CG, Silva S. Complicações na sala de recuperação anestésica, fatores de risco e intervenções de enfermagem: revisão integrativa. Rev SOBECC. 2017;22(4):218-29. http://dx.doi. org/10.5327/Z1414-4425201700040007

15. Associação Brasileira de Enfermeiros de Centro Cirúrgico, Recuperação Anestésica e Centro de Material e Esterilização (SOBECC). Diretrizes de práticas em enfermagem cirúrgica e processamento de produtos para a saúde. 7ª ed. Barueri: Manole; São Paulo: SOBECC; 2017.

16. Welte LVT, Fonseca LF. Patient recovery assessment in post-operative recovery room in absence of anesthetic. $J$ Nurs UFPE on line [Internet]. 2016 [acesso em 2016 out 25];10(6):2091-9. Disponível em: https://periodicos.ufpe. $\mathrm{br} /$ revistas/revistaenfermagem/article/view/11222/12807 DOI: 10.5205/reuol.9199-80250-1-SM1006201623

17. Volquind D, Fellini RT, Schnor OH, Flores RPG, Londero BB. Influência da escala de Aldrete e Kroulik nas estratégias de gestão da sala de recuperação pós-anestésica. Rev Adm Hosp [Internet]. 2014 [acesso em 2015 dez 8];11(4):99-104. http://dx.doi.org/10.21450/rahis.v11i1.1987

18. Silva JJA, Grossi ACMA, Haddad MDCLM, Marcon SSS. Avaliação da qualidade das anotações de enfermagem em unidade semi-intensiva. Esc Anna Nery. 2012;16(3):577-82. http://dx.doi.org/10.1590/ S1414-81452012000300021

19. Conselho Federal de Enfermagem (COFEn). Resolução COFEN n. 311/2007. Dispõe sobre o Código de Ética dos Profissionais de Enfermagem. Rio de Janeiro: COFEn; 2007 [acesso em 2015 dez 8]. Disponível em: https://www.diariodasleis.com.br/busca/exibelink. php?numlink=1-39-34-2007-02-09-311 